\title{
Title Page: Patients’ Perspectives of Bedside Nursing Handover
}

Anne McMurray PhD, RN, FRCNA, Research Chair in Nursing, Murdoch University, and Adjunct Professor, Research Centre for Clinical and Community Practice Innovation, Griffith University, Queensland, Australia

A.McMurray@murdoch.edu.au

Wendy Chaboyer PhD, RN, FRCNA, Director, Research Centre for Clinical and Community Practice Innovation, Griffith University, Queensland, Australia

Marianne Wallis, PhD, RN, FRCNA, Professor of Nursing, Gold Coast Health Service District and Research Centre for Clinical and Community Practice Innovation, Griffith University, Queensland, Australia

Joanne Johnson, MHS, RN, West Moreton South Burnett Health Services District, Ipswich Hospital, Queensland, Australia

Tanya Gehrke, RN, BN, Ipswich Hospital, Queensland, Australia

Corresponding author: Professor Anne McMurray

Email: A.McMurray@murdoch.edu.au 


\section{Acknowledgement}

Our study was funded by the Australian Commission on Safety and Quality in Health Care.

We would like to acknowledge the generosity of staff and patients at Ipswich Hospital, Queensland who gave their time and their views on bedside handover.

\section{Conflict of Interest}

none 


\title{
Patients’ Perspectives of Bedside Nursing Handover
}

\begin{abstract}
Background: Patient participation in handover is one aspect of patient-centred care, where patients are considered partners in care. Understanding the patient perspective provides a foundation for nurses to tailor their bedside handovers to reflect patients' thoughts and beliefs and encourage their active involvement in decision-making.
\end{abstract}

Aim: This study examined patients’ perspectives of participation in shift-to-shift bedside nursing handover.

Methods: A descriptive case study was conducted with ten patients in one Queensland hospital who had experienced bedside handover during their hospitalisation in 2009. Participants were asked their views about bedside handover including its benefits and limitations, their existing and potential role in handover, the role of family members, and issues related to confidentiality. Data were analysed using thematic content analysis. Findings: Four themes emerged from the analysis. First, patients appreciated being acknowledged as partners in their care. Second, they viewed bedside handover as an opportunity to amend any inaccuracies in the information being communicated. Third, some preferred passive engagement rather than being fully engaged in the handover. Fourth, most patients appreciated the inclusive approach of handover as nurse-patient interaction.

Conclusions: Bedside handover provides an opportunity for patients to be involved as active participants in their care. They value having access to information on an ongoing basis, and although not all choose the same level of interaction, they see their role as important in maintaining accuracy, which promotes safe, high quality care.

Keywords: nurse, bedside, patient, clinical handover, quality of care, communication

Word count: 4493 


\section{Competing Interests}

None 


\section{Introduction}

Clinical communication plays a critical role in patient safety, with miscommunication contributing to a large proportion of adverse events (Haig, Sutton, \& Whittington, 2006). One form of communication, shift-to-shift nursing handover, has gained research interest for three reasons. The first is a response to the worldwide safety agenda, recognising that the content and process of handover can either promote or threaten the quality of communication, and therefore safe care (former Australian Council on Safety and Quality in Health Care, 2005); Arora, Manjarrez, Dressler, Basaviah, Hatasyamani, \& Kripalani, 2009; Australian Commission on Safety and Quality in Health Care (ACSQHC), 2008; Wong, Yee, \& Turner, 2008; World Health Organization (WHO), 2008). Second, as a critical process for transferring accountability for patient care from one team of providers to another (Australian Medical Association (AMA), 2006), handover is a management tool to maintain continuity of care. Third, nursing handover conducted at the bedside provides an opportunity for engaging patients in their care, which reflects a professional commitment to patient-centred care (Chaboyer, Johnson, Hardy, McMurray, Wallis, \& Chu, 2009a; Kelly, 2005; Robinson, Callister, Berry, \& Dearing, 2008; Wiggins, 2008).

Bedside nursing handover has been the subject of a number of nursing research studies (Chaboyer et al., 2009a; Chaboyer, McMurray, \& Wallis, 2009b McKenna \& Walsh, 1997; McMurray, Chaboyer, Wallis, \& Fetherston 2009; O’Connell \& Penny, 2001). In 2009 we conducted a descriptive case study of bedside nursing handover in three wards in each of two Australian hospitals; one in Queensland and one in Western Australia (six wards in total) (Chaboyer et al., 2009b). The study was part of a national initiative by the Australian Commission for Safety and Quality in Healthcare to develop a standard operating protocol for bedside handover to minimise the threat of miscommunication-related adverse events. 
Our analysis indicated that the patient perspective was a missing element in the research. Thus, a subsequent study of patients’ perspectives of bedside handover was undertaken to better understand their role in the handover process, and the benefits and limitations of participation.

\section{Clinical handover}

Research indicates that up to two-thirds of sentinel adverse events in hospitals are related to communication problems (Haig et al., 2006). Miscommunication during clinical handover can also lead to service discontinuities, evident in unnecessary presentation to emergency departments, suboptimal patient flow through the system, readmissions, duplication of services, and patient dissatisfaction (Alem, Joseph, Kethers, Steele, \& Wilkinson, 2008; Anthony \& Hudson Barr, 2004; Bomba \& Prakash, 2005; VanWalraven, Mamdani, Fang, \& Austin, 2004). Verbal handovers at the nursing station can be unreasonably lengthy, include non-essential and irrelevant information, and may provide unreliable or inaccurate information, often focusing on subjective, speculative, sometimes vague information (Davies \& Priestly, 2006; O’Connell \& Penney, 2001; Philibert \& Leach, 2005). They can also be confined to ritualistic, retrospective, treatment oriented information (what the nurse achieved) rather than providing focus and direction for forward planning that includes information on how patients are actually coping (Cahill, 1998; Dowding, 2001; Fenton, 2006; Hopkinson, 2002; McKenna \& Walsh, 1997; Webster, 1999). Health professionals’ variable engagement with handover (Manias \& Street, 2000), and their style of communication have also played a part in miscommunication-related medical errors (IOM, 2003; Shortell \& Kaluzny, 2006). Attempts to redress these problems have led to the need for standard operating protocols for handovers (Arora et al., 2009; Botti, Buckness, Cameron, Johnstone, Redley, et al., 2009; Chaboyer et al., 2009a,b; Yee, Wong, \& Turner, 2009), ward-based whiteboards to chart 
patient status and progress, (Chaboyer et al., 2009b; Riley, Forsyth, Manias, \& Iedema, 2007), and implementation of bedside nursing handovers (Anderson \& Mangino, 2006; Broekhuis \& Veldkamp, 2007; Cahill, 1998; Chaboyer et al., 2009b; McKenna \& Walsh, 1997; McMurray et al., 2009).

\section{Bedside handover and patient-centred care}

Bedside handover was developed to improve the accuracy and timeliness of information transfer by including patient input, and adding the type of visual information that can be gleaned at the bedside (Broekhuis \& Veldkamp, 2007; Cahill, 1998). The patient-centred approach also reflects the rights of patients to be partners in their care, and the expectation that their participation in care may lead to better outcomes and greater satisfaction with care (Anderson \& Mangino, 2006; Cegala, Street, \& Clinch, 2007; Coulter \& Ellins, 2007;

Coulter, Parsons, \& Ashkam, 2008; Kravitz \& Melnikow, 2001; Robinson et al., 2008, Sidani, Epstein, \& Miranda, 2006). Sidani et al. (2006) also report that patients’ involvement in treatment-related decision making can increase their sense of control, improve functional and clinical outcomes, and reduce rates of referral and diagnostic test reordering.

Despite the attraction of a patient-centred approach there are a number of contentious issues related to bedside handover. Some claim that it is time and resource intensive (AMA, 2006; Cahill, 1998), and that clinical jargon may be disturbing or dehumanising to patients (Cahill, 1998; Martin, Greenhouse, Merryman, Shovel, Liberi, \& Konzier, 2007; Rutherford,, Lee \& Greiner, 2004). Studies also report a fear by nurses, of breaching patient confidentiality (Greaves, 1999). Cahill’s (1998) study of ten surgical patients’ opinions of bedside handover found that confidential disclosure was not a concern for nine of the ten informants. Instead, their concerns surrounded nurses maintaining professional distance between themselves and the patients, a lack of comprehensiveness of information, and the 
need for handovers to ensure their physical and psychological safety (Cahill, 1998). Greaves (1999) interviewed four patients, and found that they wanted greater involvement, access to information and assurance of continuity of care. As with Cahill's (1998) study, issues related to confidentiality were not an overriding concern, with participants reporting that it was easy to just 'shut out' conversations about others. Two more recent studies in the UK found that patients were satisfied with bedside handover, and, as in Cahill’s (1998), and Greaves’ (1999) studies, they had no concerns about confidentiality (Kassean \& Jagoo, 2005; Kelly, 2005)

The lack of widespread use of bedside handover may be due to the structure and function of most practice settings, which reinforce the expert model of practice rather than partnerships between nurses and patients (Brown et al., 2006, Gallant et al., 2002). Gallant et al.’s (2002) concept analysis of nurse-patient partnership revealed that the term has been associated with collaboration, participation, mutuality, and citizen involvement. They report that a shift from the traditional, hierarchical, expert model of practice to a more patientenabling, participative approach, has created an empowering environment for the patient (Gallant et al., 2002). In this context, the nurse is engaged with patients as a reflexive, critical listener to build a trusting relationship (Brown, McWilliam, \& Ward-Griffin, 2006). Patientcentred care is therefore patient-empowering, with patients choosing the extent of their involvement in care according to their knowledge, abilities, preferences and rights (Brown et al., 2006).

Hook’s (2004) review of the literature on patient partnerships confirmed nurses' professional valuing of the empowering attributes defined by Gallant et al. (2002), including shared decision-making and patient autonomy. However, her analysis revealed an absence of theoretical connections between the concept of partnership and the delivery of nursing care 
(Hook, 2004). To advance this body of knowledge Hook (2004) recommended evaluating clinical situations that would benefit from partnerships.

To date, nursing research into partnerships and patient-centredness has focused on partnerships in community settings (Aston, Meagher-Stewart, Edwards, \& Young, 2009; Bruni, Laupacis, \& Martin, 2008) or in home care or aged care (Brown et al., 2006; Pajnkihar, 2009). One Australian pilot study on patient-centred care analysed influences on and barriers to developing partnerships, confirming communication as the greatest barrier to genuine partnerships (Keatinge, Bellchambers, Bujack, Cholowski, Conway, \& Neal, 2002). Pajnkihar's (2009) study also canvassed the views of nurse leaders on partnerships, revealing that although nurses declare support for active involvement of patients and families, practice remains focused on standardised routines, predictable patterns and professional hierarchies. In Australia, the patient focus is also being discussed as part of a national safety and quality improvement strategy (Baggoley, Curtis, Dunbar, \& Jorm, 2009). The research reported in this paper contributes one increment in advancing this body of knowledge. It is based on the contention that patient perceptions are the most reliable measure of patient-centredness (Robinson et al., 2006).

\section{Aim}

The aim of the study was to interpret patients' perceptions of shift-to-shift bedside handover in nursing. The findings were intended to provide a foundation for tailoring nursing bedside handovers to better reflect patients' thoughts and beliefs, and encourage their active involvement in decision-making.

\section{Methodology}


This descriptive case study explored Australian patients’ perspectives of bedside handover. Case study, which is a pervasive approach in nursing research (Anthony \& Jack, 2009), is typically an idiographic, intensive study of a single case for the purpose of understanding a larger class of similar units (Yin, 2003). The basic goal of case study method is to analyse a case bounded by a definable context, which in this case, was the situation of bedside nursing handover. Interview data from participants were analysed in terms of relational processes, the context, the issues of concern, and the various situations unique to each. As Yin (2003) suggests, each unit of analysis is contextualised to features of the specific situation, then subunits of data are integrated in the overall case to illuminate and compare both unique and common findings. This involved iterative content analysis of individual interviews, and ongoing comparative analysis of responses from other participants. In seeking convergence of findings from the analysis, there was no expectation of generaliseable findings, but rather an intention to extrapolate meaningful information that could be useful in other settings (Yin, 2003).

\section{Participants}

A convenience sample of ten patients admitted to one of two medical units in one Queensland hospital was recruited during 2009. Inclusion criteria included English speakers having been hospitalised on the ward for at least overnight, and able to tolerate a 30-60 minute interview. Patients who were critically ill or infectious or those unable to consent were excluded. Patients were approached by the nurse unit managers (NUMs) or their designates on each ward and asked to volunteer for the study.

\section{Data collection}

The NUMs identified all patients who had agreed to participate to the three researchers conducting the interviews (AM, TG, WC). Interview appointments were made at a time 
convenient to the patient and held in a private office on the ward in all but two cases, which were conducted at the bedside because of a lack of patient mobility. In all cases privacy was maintained without interruption. Interviews were audio-taped, semi-structured, about 3/4 to 1 hour in duration, prompted by the following:

1. What do you think about nurses undertaking their shift to shift handover at your bedside?

2. From your perspective, what are some of the benefits of bedside handover?

3. What are some of the limitations of bedside handover?

4. What do you think your role as a patient is in the bedside handover (i.e. how do you currently participate)?

5. What do you think your role as a patient could be in the bedside handover (i.e. how do you think you should/could participate)?

6. What role do you think your family members might have in bedside handover?

7. To what extent to you think that bedside handover compromises your privacy or confidentiality (please explain)?

8. Are there topics you think should be excluded from the bedside handover? If so, what are they?

9. Is there any extra information you think should be included in the handover that would help you and your family once you are discharged home?

\section{Ethical considerations}

Ethics approval was granted by the University and the Health Service District. Potential participants were informed about the study verbally by the NUM. They were given a written information summary sheet by the interviewer prior to the interview, explaining the aim of the study and how the information would be used. Written consent was obtained after all individuals were given assurances of continued care regardless of participation, 
confidentiality, and the right of refusal to answer any questions and to withdraw from the study at any time.

\section{Data analysis}

Interviews were tape recorded, transcribed, and analysed using thematic content analysis. Analysis was iterative, including line by line analysis of transcripts, refining emerging codes into themes or units of meaning, which were then compared and coded through pattern matching (DeSantis \& Ugarizza, 2000; Miles \& Huberman, 1994). Themes and relationships were re-examined and recoded by two members of the research team (AM, WC) in a recursive manner as follows: First level coding involved reading responses to locate the major similarities or themes. A second level of analysis coded the major themes into subcategories and relationships. A third level further analysed the organisation of themes, categories of response, and relationships to examine the data for consistency (Miles \& Huberman, 1994; Patton, 2002). Analysis continued until no new themes emerged and there was agreement on themes.

\section{Validity and reliability}

Trustworthiness of the data was maintained by appropriate sample selection to ensure credibility, and by creating an audit trail to illustrate systematic documentation and show the logic flow of the data collection and analysis (Lincoln \& Guba, 1985). Because of the transience of the patient population we were unable to conduct member checks to verify the findings with the participants as a way of completing our argument for fittingness, or transferability of findings. Instead, we conducted a consensus conference between all members of the research team to peruse our initial findings and confirm their meaningfulness with respect to ongoing feedback from the two wards where bedside handover is the norm.

\section{Findings}


Participants included six females and four males ranging in age from 52 to 74 years (median 68 years). They had been hospitalised on the study wards for 1-17 days (median 4 days), so all had experienced bedside handover. Four main themes emerged from the data: 'acknowledging patients as partners', 'amending inaccuracies', 'passive engagement', and 'handover as interaction'.

\section{Acknowledging patients as partners}

Being acknowledged as a partner reflected patients’ perceptions that by sharing professional information, nurses were recognising that patients were knowledgeable and had a legitimate right to information on their condition. This made them feel that care was personalised and that they were a person first, and a patient second, particularly when they were introduced at handover. They believed this helped them know how their care was progressing, and gave them an indication of who would be caring for them in the upcoming shift.

Typical statements included the following:

"You do get the feeling of at least being wanted, you're not just a patient in a bed...it's better than the way doctors used to do their rounds - the ease with which it's done, in simple language....”

"They don't introduce you as a patient or a number, which...alienates you straight away. They actually say, 'this is [name] and [name's] problems are this. So it actually brings you involvement with the oncoming staff."

“...it makes you feel like you're involved...like at the nurses' station they wouldn't have a clue who they were talking to or about."

\section{Amending inaccuracies}

Participants had a common view that the handover was an opportunity for them to gain information about their medical condition, and to understand staff expectations for their progress, and upcoming plans for their care. Some believed that their role was to ensure the 
accuracy of information being exchanged, such as whether or not the doctor had visited, or medications or treatments had been given. A number of comments illustrate this.

"If they got anything wrong you could always put them right...if they explain that you're on insulin you can tell them that you're on two lots of insulin...and oh yes, but I'm on Humarsol as well."

"I actually had to correct them this morning. I had my drain in and the nurse said 'one' and I said 'no, four'!”

One patient explained that she was quite forthright in correcting any misinformation being transmitted at handover.

“...there may be somebody coming [on shift] who doesn't know anything about you and doesn't know... what needs you have or anything else, so I think it's a good thing. Also, a number of times in here I'll say 'that's not right, that didn't happen you know, you've got it all wrong there.",

\section{Passive engagement}

Some participants preferred a less participative approach, seeing their role as passive listeners. They paid attention to the exchange of information between nurses but did not contribute information unless they were expressly invited. One suggested that when staff members talked in the third person, it meant they were not encouraging patients' input. "The information depends on who is giving the handover. They could be more inclusive." However, others simply wanted to gather whatever information they could from what were relatively short handovers (1-3 minutes).

"I think you should just be there and just listen."

"...it would be rude, the height of ignorance because they're doing their job the way they've been taught and then I'm interfering aren't I?"

\section{Handover as interaction}

In many cases the nurses handing over actively encouraged patient engagement. Participants thought this was a more inclusive approach, especially those who declared they wanted to know everything about their condition. They were reportedly pleased to be asked for input, especially when this helped clarify their expectations or misunderstandings. 
"In my case the nurses know I like to enter into the discussion and they invite me to ask questions."

"Well, you let them know where you stand...it might just be by the manner you look at them or the way you sit. I think every patient does give body language...one way or another, so it lets the staff...know basically what you are about."

“Well you're the subject aren't you? I mean you're the topic of conversation in the handover...they're talking about you so if you've got anything to say by all means say it and...they can sort of discuss it. You can get involved, you can speak to them and get involved in it."

Although this was a typical perception among participants, one person did not wish to interact, and another felt there was no encouragement to interact during handover because there was no invitation to ask questions. Another man commented that he would have liked to have discussed his diagnosis of heart failure at handover. However, like most others, he understood the time pressures of handover, which was a common limitation identified by participants.

We had expected that confidentiality of information and privacy would be an issue for most of the patients. However, only two expressed any concern about the sensitivity of information being shared in a four bed room. Their comments were not about the handover process, but rather reflected issues about having both males and females in the same room. The others were unequivocal that it was not an issue.

"They have never yet been invasive of my privacy...they are not delving into private parts of my illness or parts of anything else, you know. They're just more or less passing on the information that I'm a COPD person.”

Participants indicated that the language of communication was appropriate, which may be a feature of the clinical environment, as reflected in the following comment.

"I can remember a few years ago the doctors used to do their rounds and talk to their subordinates as if you weren't there...the bedside manner of the nurses is totally an entirely different thing."

\section{Discussion}


Because data collection was confined to a convenience sample the study findings are not generaliseable. Further limitations include the lack of validity and generalisability, which is due to the small sample size. However, the analysis provides a useful insight into the way patients perceive bedside handover. Despite the single Australian context, the similarities in clinical care in other settings suggest a level of applicability elsewhere. These findings exemplify the type of issues that may arise in other institutions changing to bedside handover.

Our findings reflect a move to patient-centred care in the hospital where the study was undertaken. Patient-centred care represents one 'pillar' of a broader Transforming Care At the Bedside initiative, which had been introduced at the study site three years prior to the study (Chaboyer et al., 2009a; Rutherford et al., 2004). Clearly, patients appreciated this refocusing of clinical practice. It was interesting to find that, like earlier studies of patients' perceptions of bedside handovers (Cahill, 1998; Greaves 1999; Kassean \& Jagoo, 2005; Kelly, 2005), patients were not overly concerned with privacy. Instead, they all expressed a desire to be involved in handover and, as such, have access to information. This suggests that privacy of health-related information may be more of an issue to nurses than patients. Participants' comments reflected a desire to be engaged with nursing staff, to be seen as a partner in their care.

A recent systematic review of bedside handover conducted by Arora et al., (2009) found that including the patient can be empowering, which is one of the goals of contemporary nursing practice (Robinson et al., 2006). This represents a shift in the nursepatient relationship over the past decades. Almost two decades ago, Australian research into bedside handover concluded that it was an inefficient use of time, as handover consisted of a simple recitation of facts (Parker, Gardner, \& Wiltshire, 1992). Our study revealed a perception that nurses were more inclusive in their handovers, moving beyond a mere status 
report, and making a conscious effort to engage patients. This resonates with a suggestion by Burnard (1987) that a more comfortable patient-centred atmosphere would actually increase the effectiveness of bedside handover. It also challenges the expert model of practice, instead supporting the groundswell of nursing research recommending power sharing and nursepatient partnerships (Anderson \& Mangino, 2006, Brown et al., 2006; Gallant et al., 2002; Keatinge et al., 2002; Robinson et al., 2008; Wiggins, 2008). A parallel movement is also gaining popularity in medical circles on the basis that closer alignment with patient preferences not only improves satisfaction with care but enhances adherence with treatment (Cegala et al., 2007; Weston, 2001; Young, 2008). International trends in health service delivery also reflect a change to patient-centredness and a partnership approach to care (Baggoley et al., 2009; Bruni et al., 2008; Coulter et al., 2008). In fact, the Australian Commission on Safety and Quality in Health Care identifies patient-centred services as central to clinical redesign strategies and the development of trust between service providers and patients (ACSQHC, 2009).

Our findings are also consonant with Anderson and Mangino’s (2006) findings identifying information (how well the nurses kept you informed) as the most significant element in patient satisfaction with bedside handover. The structures and processes of bedside handover, articulated in our standardised operating protocol, included a number of steps to ensure congruence and comprehensiveness of information (Chaboyer et al., 2009b). These included a safety scan at the bedside, integrating information from the handover guide, which was updated regularly throughout the shift, and access to clinical information contained in the bedside patient chart. Clearly demonstrating to the patient how all of this information was used in care planning may have added to their reassurance and therefore their perceptions of accuracy and safety. 
The style of communication used by the nurses was mentioned as a positive aspect of bedside handover. Patients believed that by using language familiar to them, nurses were shifting to a more mutually trusting relationship, sharing knowledge. Clearly, when patients are given an opportunity to gain a greater understanding of their plan of care they will be better equipped for input into collaborative decisions aimed at ensuring that their care is both appropriate and safe. As Williams (2002) suggests, one of nursing's major goals should be decision-making at the point where knowledge is greatest, and this requires an organisational culture where staff empowerment becomes an antecedent to patient empowerment. From this type of synergy there is the potential to advance both patient outcomes and clinical development (Williams, 2002).

The interactive nature of bedside handover was another feature of the style of communication. A non-linear, transactional approach to communicating reflects a process in which messages are sent and received simultaneously (Adler \& Rodman, 2009), and where participants are constantly and mutually influencing each other (Miller, 2002). Patients were active participants in the handover, and were able to not only contribute information, but also correct any inaccuracies or misinformation. What was not achieved in these bedside handovers was the opportunity to discuss psychological and social information, and this was a shortcoming found in other research (Cahill, 1998; Dowding, 2001; Fenton, 2006; Hopkinson, 2002; McKenna \& Walsh, 1997; Webster, 1999) and in a recent report of the Special Commission into public hospitals in New South Wales Australia (Garling, 2008). Increased time pressures on a workforce that is struggling to cope with staff shortages make it highly unlikely that this situation will change (Preston, 2009). 


\section{Conclusions}

This analysis demonstrated that bedside nursing handover is an ideal vehicle for implementing a partnership model of care. Patients believed they were being treated in a trusting, respectful way, and felt welcome to correct any inaccuracies. They saw handover conducted at their bedside as an opportunity for interaction, even though some adopted a role of passive engagement. Although the study was confined to a single group of patients in one setting it was clear that patients perceived bedside handover as a tool to promote accurate communication. This type of communication strategy is a fundamental building block for patient empowerment, which is the essence of nurse-patient partnerships in a contemporary model of care.

The findings suggest a number of recommendations for practice, management and further research. First, clinical areas should consider the adoption of bedside handover as a mechanism to promote patient centred care and as a patient safety measure. Second, there is a need for ongoing development of communication skills. Although these skills are part of undergraduate curricula, communication is not always considered a priority in orientation and staff development programs. Content would include an emphasis on introducing patients at handover, being inclusive in the context of transmitting information, and assessing whether or not a patient wishes to be actively or passively involved in their handover. For managers the implementation of bedside handover should be incorporated in a change management strategy that is collaborative to facilitate quality improvement. Managers and staff development personnel should also be aware of the need for staff to discuss the changes, to

ensure a level of comfort with disclosing information that may have previously been withheld from patients. This should also include addressing nurses' concerns about confidentiality and justifying the need for a greater focus on patient-centred care and its benefits. 
The study suggests a need for further research into other elements of Transforming Care At the Bedside to determine which elements of this approach are most effective, and the best methods of implementation. Ongoing research is also needed into strategies that foster patient-centred care, and the relationship of different types of information and patient decision-making. Additional studies should address barriers and facilitating factors influencing the adoption of bedside handover and other aspects of patient-centred care with the ultimate aim of providing robust evidence for policy and practice change. 


\section{References}

Adler, R. B., \& Rodman, G. (2009) Human communication: what and why. In Understanding Human Communication, $10^{\text {th }}$ edition,). New York: Oxford University Press,1-9.

Alem, L., Joseph, M., Kethers, S., Steele, C., \& Wilkinson, R. (2008). Information environments for supporting consistent registrar medical handover. Health Information Management Journal, 37(1), 9-25.

Anderson, C., \& Mangino, R. (2006). Nurse shift report: who says you can't talk in front of the patient? Nursing Administration Quarterly, 30(2),112-122.

Anthony, M., \& Hudson-Barr, D. (2004). A patient-centered model of care for hospital discharge. Clinical Nursing Research, 13(2), 117-136.

Anthony, S., \& Jack, S. (2009). Qualitative case study methodology in nursing research: an integrative review. Journal of Advanced Nursing, 65(6), 1171-1181.

Arora, V., Manjarrez, E., Dressler, D., Basaviah, P., Hatasyamani, L., \& Kripalani, S. (2009). Hospitalist handoffs: A systematic review and task force recommendations. Journal of Hospital Medicine 4(7), 433-440.

Aston, M., Meagher-Stewart, D., Edwards, N., \& Young, L. (2009). Public health nurses' primary health care practice: strategies for fostering citizen participation. Journal of Community Health Nursing 26, 24-34.

Australian Council for Safety and Quality in Health Care (2005). Clinical Handover and Patient Safety, Pub. No. 3640, Sydney: Australian Resource Centre for Healthcare Innovations.

Australian Commission on Safety and Quality in Health Care. (2008). Nine priority programs. Available from URL: http://www.safetyandquality.gov.au (Accessed 16 March 2009).

Australian Commission on Safety and Quality in Healthcare (2009). Discussion Paper on achieving the directions established in the proposed national safety and quality framework. Sydney: ACSQHC.

Australian Medical Association (AMA) (2006). Safe handover: Safe patients; guidelines on clinical handover for clinicians and managers. http://www.ama.com.au/web.nsf/doc/WEEN6XFDKN/pdf (accessed March 10, 2009).

Baggoley, C., Curtis, I., Dunbar, N., \& Jorm, C. (2009). A conversation about health care safety and quality. Medical Journal of Australia, 191(1),7-8.

Bomba, D., \& Prakash, R. (2005). A description of handover processes in an Australian public hospital. Australian Health Review, 29(1), 68-79. 
Botti, M., Bucknall, T., Cameron, P., Johnstone, M-J., Redley, B., Evans, S., \& Jeffcott, S. (2009). Examining communication and team performance during clinical handover in a complex environment: The private sector post-anaesthetic care unit. Medical Journal of Australia 190 (Suppl), s157-s160.

Broekhuis, M., \& Veldkamp, C. (2007). The usefulness and feasibility of a reflexivity method to improve clinical handover. Journal of Evaluation in Clinical Practice, 13, 109115.

Brown, D., McWilliam, C., \& Ward-Griffin, C. (2006). Client-centred empowering partnering in nursing. Journal of Advanced Nursing, 53 (2), 160-168.

Bruni, R., Laupacis, A., \& Martin, D. (2008). Public engagement in setting priorities in health care. Canadian Medical Association Journal, 179(1), 15-18.

Burnard, P. (1987). Meaningful dialogue. Nursing Times, 83 (20), 43-45.

Cahill, J. (1998). Patient's perceptions of bedside handovers. Journal of Clinical Nursing, 7, 351-359.

Cegala, D., Street, R., \& Clinch, C. (2007). The impact of patient participation on physicians' information provision during a primary care medical interview. Health Communication, 2(2), 177-185.

Chaboyer, W., Johnson, J., Hardy, L., McMurray, A., Wallis, M., \& Chu, S. (2009a). Bedside handover: Quality improvement strategy to 'Transform Care at the Bedside'. Journal of Nursing Care Quality, 24(2), 136-142.

Chaboyer, W., McMurray, A., \& Wallis, M. (2009b). Communicating patient information: Structures, Processes and Outcomes of Bedside Handover in Nursing. International Journal of Nursing Practice, in press.

Chin, H., \& McNichols, E. (2000). Practice development credentialing in the United Kingdom: A unique framework for providing excellence, accountability, and quality in nursing and healthcare. On-line Journal of Issues in Nursing, 5 (2), 17-22.

Coulter, A., \& Ellins, J. (2007). Effectiveness of strategies for informing, educating, and involving patients. British Medical Journal, 335, 24-27.

Coulter, A., Parsons, S., \& Ashkham, J. (2008). Where are the patients in decision-making about their own care? Policy Brief, WHO and WHO European Observatory on Health Systems and Policies. Copenhagen: Regional Office for Europe.

Davies, S., \& Priestley, M.J. (2006). A reflective evaluation of patient handover practices. Nursing Standard, 20, 49-52.

DeSantis, L., \& Ugarriza, D. (2000). The concept of theme as used in qualitative nursing research, Western Journal of Nursing Research, 22(3), 351-372. 
Dowding, D. (2001). Examining the effects that manipulating information given in the change of shift report has on nurses' care planning ability. Journal of Advanced Nursing, 33 (6), 836-846.

Fenton, W. (2006). Developing a guide to improve the quality of nurses' handover. Nursing Older People, 18(11),32-36.

Gallant, M., Beaulier M., \& Carnevale, F. (2002). Partnership: an analysis of the concept within the nurse-client relationship. Journal of Advanced Nursing, 40 (2), 149-157.

Garling, P. (2008). Final Report of the Special Commission of Inquiry: Acute Care in NSW Public Hospitals, 2008 - Volume 2. Sydney: State of NSW.

Greaves, C. (1999). Patients’ perceptions of bedside handover. Nursing Standard, 14(12), 3235.

Haig, K.M., Sutton, S., \& Whittington, J. (2006). SBAR: a shared mental model for improving communication between clinicians. Joint Commission's Perspectives on Patient Safety, 32, 167-175.

Heath, R. L., \& Bryant, J. (2000). Human Communication Theory And Research: Concepts, Contexts, and Challenges, $2^{\text {nd }}$ edition. New Jersey: Lawrence Erlbaum Associates.

Hopkinson, J. (2002). The hidden benefit: the supportive function of the nursing handover for qualified nurses caring for dying people in hospital. Journal of Clinical Nursing, 11:16-175.

Hook, M. (2004). Partnering with patients - a concept ready for action. Journal of Advanced Nursing, 56 (2), 133-143.

Institute of Medicine (2003). Keeping patients safe: transforming the environment of nurses. Washington DC: National Academics Press.

Keatinge, D., Bellchambers, H., Bujack, E., Cholowski, K., Conway, J., \& Neal, P. (2002). Communication: Principal barrier to nurse-consumer partnerships. International Journal of Nursing Practice, 8, 16-22.

Kelly, M. (2005). Change from an office-based to a walk-around handover system. Nursing Times, 101(10), 34-35.

Kravitz, R., \& Melnikow, J. (2001). Engaging patients in medical decision making. British Medical Journal, 323, 584-5.

Lincoln, Y., \& Guba, E. (1985) .Naturalistic Inquiry. Beverley Hills, CA: Sage Publications.

Manias, E., \& Street A. (2000). Possibilities for critical social theory and Foucault's work: a toolbox approach. Nursing Inquiry, 7(1), 50-60.

McMurray, A., Chaboyer, W., Wallis, M., \& Fetherston, C. (2009). Implementing bedside handover: Strategies for change management. Journal of Clinical Nursing, in press. 
Martin, S.C., Greenhous,e P.K., Merryman, T., Shovel J., Liberi, C.A., \& Konzier, J. (2007). Transforming care at the bedside: implementation and spread model for single-hospital and multihospital systems. Journal of Nursing Administration, 37, 444-451.

McKenna, L., \& Walsh, K . (1997). Changing handover practices: One private hospital's experiences. International Journal of Nursing Practice, 3, 128-132.

Miles, M., \& Huberman, A (1994). An Expanding Sourcebook: Qualitative Data Analysis, $2^{\text {nd }}$ edition, Thousand Oaks, CA: Sage Publications.

Miller, K. (2002). Conceptual foundations: What is communication? In Communication Theories: Perspectives, Processes, and Contexts. Boston: McGraw-Hill Companies Inc., 217.

O'Connell, B.,\& Penney, W. (2001). Challenging the handover ritual. Recommendations for research and practice. The Collegian, 8, 14-18.

Pajnkihar, M. (2009). Nurses' (un) partner-like relationships with clients. Nursing Ethics 16 (1), 43-56.

Patton, M. Q. (2002). Qualitative Research and Evaluation Methods, $3^{\text {rd }}$ edn. Thousand Oaks, CA: Sage Publications,.

Parker, J., Gardner, G., \& Wiltshire, J. (1992). Handover: the collective narrative of nursing practice. Australian Journal of Advanced Nursing, 9, 31-37.

Philibert, I., \& Leach, D. (2005). Reframing continuity of care for this century. Editorial, Quality and Safety Health Care, 14, 394-396.

Preston, B. (2009). The Australian nurse and midwifery workforce: Issues, developments and the future. Collegian 16,3-9.

Riley, R., Forsyth, R., Manias, E., \& Iedema, R. (2007) Whiteboards: Mediating professional tensions in clinical practice. Communication \& Medicine, 4, 165-175.

Robinson, J., Callister, L., Berry, J., \& Dearing, K. (2008) Patient-centered care and adherence: Definitions and applications to improve outcomes. Journal of the American Academy of Nurse Practitioners, 20, 600-607.

Rutherford, P., Lee, B., \& Greiner, A. (2004) Transforming care at the bedside. Cambridge, Ma: Institute for Healthcare Innovations.

Sidani, S., Epstein, D., \& Miranda, J. (2006). Eliciting patient treatment preferences: A strategy to integrate evidence-based and patient-centered care. Worldviews on EvidenceBased Nursing, 3 (3), 116-118.

Shortell, S., \& Kaluzny, A. (2006). Health Care Management, $5^{\text {th }}$ edition. New York: Thomson Delmar Learning. 
Thiede, M. (2005). Information and access to health care: is there a role for trust? Social Science \& Medicine, 61, 1452-1462.

VanWalraven, C., Mamdani, M., Fang, J., \& Austin, P. (2004). Continuity of care and patient outcomes after hospital discharge, Journal of General Internal Medicine, 19, 624-631.

Webster, J. (1999). Practitioner-centred research: an evaluation of the implementation of the bedside hand-over. Journal of Advanced Nursing, 30(6), 1375-1382.

Wiggins, M. (2008). The partnership care delivery model: an examination of the core concept and the need for a new model of care. Journal of Nursing Management, 16, 629-638.

Williams, T. (2002). Patient empowerment and ethical decision making: the patient/partner and the right to act. Dimensions of Critical Care Nursing, 21, 100-104.

Wong, MC., Yee, KC., \& Turner, P. (2008). Clinical handover literature review. eHealth Services Research Group, Launceston Australia: University of Tasmania.

World Health Organization (2007). Communication during patient handovers. Patient Safety Solutions 1(3), Available from: http://www/who.int/patientsafety, Accessed 26 May, 2008.

Yee, KC., Wong, MC., \& Turner, P. (2009) ."Hand me an isobar”: a pilot study of an evidence-based approach to improving shift-to-shift clinical handover. Medical Journal of Australia, 190 (11), S121-S124.

Yin, R. (2003). Case Study Research Design and Methods, $3^{\text {rd }}$ edition. Thousand Oaks, CA: Sage Publications.

Young, R. (2008). Who's afraid of the empowered patient? Journal of the American Medical Association, 300 (12), 1393-1394. 